\title{
Antenatal steroids are associated with a reduction in the incidence of cerebral white matter lesions in very low birthweight infants
}

\author{
R Agarwal, M L Chiswick, S Rimmer, G M Taylor, R J Q McNally, R D Alston, \\ S W D'Souza
}

See end of article for authors' affiliations

.......................

Correspondence to: Dr D'Souza, Academic Unit of Child Health,

University of Manchester,

St Mary's Hospital,

Hathersage Road,

Manchester M13 OJH, UK;

sdesouza@man.ac.uk

Accepted

30 October 2001

\begin{abstract}
Aims: To investigate whether antenatal steroids reduce the incidence of cerebral white matter lesions in very low birthweight infants.

Methods: A total of 224 newborn infants of $<31$ weeks gestational age and weighing < $1500 \mathrm{~g}$ was studied between January 1998 and June 2000. Obstetric and neonatal information was obtained from the case notes. The study population was subdivided into two groups according to antenatal steroid exposure. A complete course of treatment consisted of two doses of $12 \mathrm{mg}$ each of betamethasone given at an interval of 12-24 hours. Infants in group 1 were born to mothers who had not received betamethasone, or were delivered within 24 hours of receiving the first dose of steroid. Infants in group 2 were born to mothers who had received one or more complete courses of betamethasone and were delivered $>24$ hours after receiving the first dose of steroid.

Results: The two groups contained statistically similar proportions of boys and girls, and the infants had similar birth weights and survival rates. Those in group 2, compared with those in group 1, had a lower gestational age $(p=0.02)$ and a lower incidence of white matter lesions on cranial ultrasound scans $(p=0.03)$. Stepwise logistic regression analysis showed that gestational age $(p=0.0002)$ and a complete course of antenatal steroids $(p=0.02)$ had independent effects on cerebral white matter lesions.

Conclusions: These observations suggest that a complete course of antenatal steroids may have a protective effect against cerebral white matter lesions in very low birthweight infants.
\end{abstract}

$\mathrm{S}$ nce the introduction of antenatal steroids in the 1970s for women at risk of preterm delivery, the incidence and severity of respiratory distress syndrome in the newborn has decreased. ${ }^{12}$ Furthermore, antenatal steroids may reduce other conditions caused by prematurity, including necrotising enterocolitis and patent ductus arteriosus. ${ }^{3}{ }^{4}$ A meta-analysis of several studies by Crowley et $a^{2}$ has shown that antenatal steroids also reduce the risk of intracranial haemorrhages in preterm infants. More recently, Leviton et al reported a reduced risk of ventriculomegaly and echolucency in cranial ultrasound scans of preterm infants whose mothers had received steroids antenatally.

Very low birthweight (VLBW) infants (weighing < $1500 \mathrm{~g}$ ) are at high risk of developing cerebral palsy in later childhood.$^{67}$ Abnormalities found on cranial ultrasound scans in the neonatal period are a good predictor of later outcome in such infants. Periventricular white matter lesions and persistent ventriculomegaly in particular have been associated with an adverse neurodevelopmental outcome, including developmental delay and cerebral palsy. ${ }^{8} 9$

In experimental studies, pretreatment with dexamethasone has been shown to be neuroprotective in the neonatal rat model. ${ }^{10}$ The precise mechanism of such neuroprotection is not clearly understood. It has been suggested that steroids may protect the developing brain against the effect of hypoxia-ischaemia by increasing ketone bodies, ${ }^{10}$ or protect against cytokine mediated damage. ${ }^{5}$ We therefore hypothesised that antenatal steroids may protect against cerebral white matter lesions in VLBW infants.

We investigated the effects of a complete course (or courses) of antenatal steroids on cerebral white matter lesions, diagnosed by cranial ultrasound scan, in VLBW infants admitted to our regional neonatal medical unit. Betametha- sone is routinely administered to mothers admitted to our hospital in preterm labour. A subgroup of these mothers may give birth before completing a course of antenatal steroids or, occasionally, the preterm infant is born before administration of the first dose of betamethasone. The antenatal conditions and mode of delivery in mothers who had completed one or more courses of steroids were compared with those who had not received antenatal steroids, or had failed to complete a course of steroid treatment before giving birth. We further compared outcomes in these two groups of infants to find out whether there were differences in the incidence of white matter lesions diagnosed by cranial ultrasound scan.

\section{METHODS}

All the VLBW infants of $<31$ weeks gestational age born at St Mary's Hospital, Manchester and admitted to the neonatal medical unit between January 1998 and June 2000 were included. Their mothers had either attended antenatal clinics at our hospital or were transferred in for obstetric conditions and because perinatal complications were anticipated. Gestational age was determined from an early antenatal scan or, in a small number of infants, from the mothers' last menstrual period. The latter were also assessed clinically by a consultant neonatologist who estimated the gestational age. Details of obstetric conditions and antenatal steroid administration were obtained from the obstetric case notes.

Abbreviations: VLBW, very low birthweight; PDA, patent ductus arteriosus; SEH, subependymal haemorrhage; IVH, intraventricular haemorrhage; PROM, prolonged rupture of membranes 
Table 1 Infant and maternal characteristics

\begin{tabular}{|c|c|c|c|}
\hline & $\begin{array}{l}\text { Group } 1 \text { (No/incomplete } \\
\text { course) }(\mathrm{n}=71)\end{array}$ & $\begin{array}{l}\text { Group } 2 \text { (Complete course of } \\
\text { steroids) ( } n=153)\end{array}$ & $\mathrm{p}$ Value \\
\hline \multicolumn{4}{|l|}{ Infant characteristics } \\
\hline Male & $35(49)$ & $81(53)$ & \\
\hline Female & $36(51)$ & $72(47)$ & 0.67 \\
\hline \multicolumn{4}{|l|}{ Birth weight (g) } \\
\hline 750 or less & $16(23)$ & $48(31)$ & \\
\hline $751-1250$ & 35 (49) & $78(51)$ & \\
\hline 1251 or more & $20(28)$ & 27 (18) & 0.15 \\
\hline \multicolumn{4}{|l|}{ Gestational age (weeks) } \\
\hline 25 or less & $20(28)$ & $35(23)$ & \\
\hline $26-28$ & $15(21)$ & $61(40)$ & \\
\hline 29 or more & $36(51)$ & $57(37)$ & 0.02 \\
\hline \multicolumn{4}{|l|}{ Maternal characteristics } \\
\hline \multicolumn{4}{|l|}{ Ethnicity } \\
\hline White & $56(79)$ & $120(78)$ & \\
\hline Asian & $10(14)$ & $21(14)$ & \\
\hline Afrocaribbean & $2(3)$ & $5(3)$ & \\
\hline Oriental & 1 (1) & $2(1)$ & \\
\hline Other & 2 (3) & $5(3)$ & 1.0 \\
\hline Primiparous & $26(37)$ & $30(20)$ & \\
\hline Multiparous & $45(63)$ & $123(80)$ & 0.008 \\
\hline Singleton births & $54(76)$ & $115(75)$ & \\
\hline Multiple births & $17(24)$ & $38(25)$ & 1.0 \\
\hline Pre-eclampsia & $10(14)$ & $46(30)$ & 0.012 \\
\hline Premature of the membranes & $9(13)$ & $53(35)$ & 0.0007 \\
\hline \multicolumn{4}{|l|}{ Mode of delivery } \\
\hline Vaginal delivery & $40(56)$ & $74(48)$ & \\
\hline Caesarean section & $31(44)$ & $79(52)$ & 0.32 \\
\hline
\end{tabular}

Betamethasone is the standard antenatal steroid used at our hospital. A course of treatment consisted of two doses of betamethasone, $12 \mathrm{mg}$ each, given at an interval of 12-24 hours. A further course was given after seven days, or later, at the discretion of the obstetrician if the risk of preterm delivery persisted. Some mothers had therefore received more than one course of antenatal steroids. Infants born to mothers who had received no antenatal steroids or had received steroids for $<24$ hours before delivery were classified as group 1 (no/incomplete course). The remainder were born to mothers who had received one or more courses of betamethasone $>24$ hours before delivery and were classified as group 2 (complete course of steroids).

The condition of the infant at birth, details of resuscitation, and progress in the neonatal medical unit were recorded in the case notes. Information recorded included the dose of surfactant, details of ventilator support and oxygen dependence, treatment of patent ductus arteriosus (PDA) with indomethacin, radiological evidence of necrotising enterocolitis, details of retrolental fibroplasia screening, and treatment for culture positive sepsis (in blood, urine, cerebrospinal fluid, or any other body fluid). Cranial ultrasound examination was carried out by two consultant radiologists, according to a protocol established in the neonatal medical unit for cranial ultrasonography, using an Acuson 128xP ultrasound scanner. The scans were performed using a multifrequency vector probe and scanning at a frequency of 7.0 MHz. Copies made of ultrasound images on laser imaging films were stored in the radiology department and used for later analysis. The first scan was performed shortly after birth and a further scan was carried out a week later. When clinically indicated, scans were repeated at fortnightly intervals. Scan reports by the two radiologists were reviewed independently by one of the authors (RA), a clinician, who found that they agreed with one another. Subsequently, another author (SR), a radiologist blinded to the infants' clinical details, reviewed the stored copies of the scans. The cranial ultrasound findings were classified as follows.

(1) No abnormality (normal), subependymal haemorrhage (SEH), or intraventricular haemorrhage (IVH). Ultrasound scans with "normal echogenicity" were deemed to be normal. Those with SEH (germinal layer haemorrhage) had increased echogenicity in the subependymal area within the caudothalamic region, and scans showing IVH had increased echogenicity in the ventricular system, separate from the normal choroid plexus.

(2) Ventriculomegaly. Ventricular enlargement was present on its own or in some cases with an IVH. The diagnosis was based on the ventricular index being greater than the 97th centile for postconceptional age. ${ }^{11}$ The ventricular index is defined as the distance in millimetres between the midline and the lateral border of the lateral ventricle, in the coronal plane at the level of the foramen of Munro.

(3) White matter lesions (echodense or echolucent lesions in the periventricular area). Echogenicity was recorded with respect to the degree of normality or increase in echogenicity (echodensity). The presence of echolucencies within the white matter was also recorded. The white matter was deemed to have normal echogenicity, echodense areas, or echolucent areas (cysts), but no attempt was made to infer underlying pathology or prognosis.

The parents of seven of 50 infants who had died in hospital gave permission for a full autopsy to be carried out. The findings in these postmortem reports were compared with those from the cranial ultrasound scans.

\section{Statistical methods}

Comparisons of proportions in 2 by 2 and 2 by 3 tables were made using Fisher's exact test. The proportion of infants with white matter lesions was also analysed by logistic regression. The mean surfactant dose was compared by analysis of variance. All calculations were performed in STATA (StataCorp 1999, Stata Statistical Software: Release 6.0; Stata Corporation, College Station, Texas, USA).

\section{RESULTS}

A total of 228 VLBW infants weighing $<1500 \mathrm{~g}$ with gestational ages of 24-30 weeks, born between January 1998 
Table 2 Outcome characteristics in group 1 and 2 infants

\begin{tabular}{|c|c|c|c|}
\hline Characteristics & $\begin{array}{l}\text { Group } 1 \text { (No/incomplete } \\
\text { course) }(n=71)\end{array}$ & $\begin{array}{l}\text { Group } 2 \text { (Complete course of } \\
\text { steroids) } \\
(n=153)\end{array}$ & $\mathrm{p}$ Value \\
\hline $\mathrm{O}_{2}$ dependence at 28 days & $19 / 30(63)$ & $54 / 87(62)$ & 1.0 \\
\hline $\mathrm{O}_{2}$ dependence at 36 weeks & $9 / 22(41)$ & $23 / 61(38)$ & 0.80 \\
\hline Surfactant (dose per infant) & 1.58 & 1.55 & 0.86 \\
\hline 25 weeks or less & $43 / 20(2.15)$ & $76 / 35(2.17)$ & \\
\hline $26-28$ weeks & $27 / 15(1.80)$ & $107 / 61(1.75)$ & \\
\hline 29 weeks or more & $42 / 36(1.17)$ & $54 / 57(0.95)$ & \\
\hline PDA & $18(25)$ & $30(20)$ & 0.38 \\
\hline NEC & $8(11)$ & 19 (12) & 1.0 \\
\hline ROP & $\begin{array}{l}3 \text { (12) had laser therapy of } 26 \\
\text { screened }\end{array}$ & $\begin{array}{l}5 \text { (7) had laser therapy of } 67 \\
\text { screened }\end{array}$ & 0.68 \\
\hline Sepsis & $29(41)$ & $64(42)$ & 1.0 \\
\hline Mortality & $20(28)$ & $30(20)$ & 0.17 \\
\hline Cranial ultrasound scans & & (1 not done) & \\
\hline Normal/SEH/IVH & 49 (69) & $123(81)$ & \\
\hline Ventriculomegaly & $4(6)$ & $9(6)$ & \\
\hline White matter lesions & $18(25)$ & $20(13)$ & 0.03 \\
\hline $\begin{array}{l}\text { Survivors with white matter } \\
\text { lesions }\end{array}$ & $7 / 51(14)$ & $15 / 123(12)$ & 0.81 \\
\hline
\end{tabular}

and June 2000, were admitted to the neonatal medical unit. Four infants had major congenital abnormalities (trisomy 18, myotonic dystrophy, holoprosencephaly, and restrictive dermopathy) and were excluded from the study. The remaining 224 infants included $71(32 \%)$ in group 1 and $153(68 \%)$ infants in group 2. Most of the mothers of the infants in group 2 had received one to three courses of steroid treatment, but a few had received four to seven courses.

Table 1 shows the clinical characteristics of these 224 infants and their mothers. Most infant and maternal characteristics recorded were similar in group 1 and group 2 apart from a higher incidence of multiparous women $(p=0.008)$, pre-eclampsia $(\mathrm{p}=0.012)$, prolonged rupture of membranes (PROM; rupture of membranes for $>24$ hours; $p=0.0007$ ), and a different gestational age distribution $(\mathrm{p}=0.02)$ in group 2. PROM, in particular, had occurred about three times more often in group 2 than in group 1.

Table 2 shows the progress of both groups of infants in the neonatal medical unit and details of their cranial ultrasound findings. Infants in the two groups had a statistically similar incidence of chronic lung disease, based on oxygen dependence at either 28 days of life or 36 weeks postconceptional age. There was little difference in the surfactant requirement in each group. All the infants born at 28 weeks of gestational age

\begin{tabular}{|c|c|c|c|}
\hline \multicolumn{4}{|c|}{ Univariate analysis of conditions associated with cerebral white matter } \\
\hline Variables & $\begin{array}{l}\text { White matter lesions } \\
(\mathrm{n}=38)\end{array}$ & $\begin{array}{l}\text { No white matter lesions } \\
(n=185)\end{array}$ & $\mathrm{p}$ Value \\
\hline Male & $19(50)$ & $96(52)$ & \\
\hline Female & $19(50)$ & $89(48)$ & 0.86 \\
\hline \multicolumn{4}{|l|}{ Gestational age (weeks) } \\
\hline$\leqslant 25$ & $18(47)$ & $36(19)$ & \\
\hline $26-28$ & $13(34)$ & $63(34)$ & \\
\hline$\geqslant 29$ & $7(18)$ & $86(46)$ & 0.0004 \\
\hline \multicolumn{4}{|l|}{ Birth weight (g) } \\
\hline$\leqslant 750^{\circ}$ & $18(47)$ & $45(24)$ & \\
\hline $750-1250$ & $17(45)$ & $96(52)$ & \\
\hline$\geqslant 1251$ & $3(8)$ & $44(24)$ & 0.007 \\
\hline \multicolumn{4}{|l|}{ Antenatal steroids } \\
\hline No/incomplete steroids & $18(47)$ & $53(29)$ & \\
\hline Complete course(s) & $20(53)$ & $132(71)$ & 0.03 \\
\hline Pre-eclampsia & $8(21)$ & $48(26)$ & 0.68 \\
\hline \multicolumn{4}{|l|}{ Mode of delivery } \\
\hline Vaginal & $24(63)$ & $90(49)$ & \\
\hline Caesarean section & $14(37)$ & $95(51)$ & 0.11 \\
\hline \multicolumn{4}{|l|}{ Duration of PROM } \\
\hline$\leqslant 24$ hours & $15 / 22(68)$ & $58 / 113(51)$ & \\
\hline$>24$ hours & $7 / 22(32)$ & $55 / 113(49)$ & 0.17 \\
\hline PDA requiring indomethacin & $14(37)$ & $34(18)$ & 0.017 \\
\hline Sepsis & $20(53)$ & 73 (39) & 0.15 \\
\hline Survivors & $22(58)$ & $152(82)$ & 0.002 \\
\hline \multicolumn{4}{|l|}{ CRIB score } \\
\hline $0-5$ & $19(50)$ & $136(74)$ & \\
\hline $6-10$ & $13(34)$ & $31(17)$ & \\
\hline $11-15$ & $5(13)$ & 17 (9) & \\
\hline 16 or more & $1(3)$ & 1 (1) & 0.015 \\
\hline
\end{tabular}


Table 4 Effect of antenatal steroids on cerebral white matter lesions (WML)

\begin{tabular}{llllll}
\hline \multirow{2}{*}{$\begin{array}{l}\text { Gestational age } \\
\text { (weeks) }\end{array}$} & \multicolumn{2}{l}{ Group 1 (No/incomplete course) } & & \multicolumn{2}{l}{ Group 2 (Complete course of steroids) } \\
\cline { 2 - 3 } \cline { 5 - 6 } & All infants & No with WML & & All infants & No with WML \\
\hline 525 & 20 & $10(50)$ & $4(27)$ & 34 & $8(24)$ \\
$26-28$ & 15 & $4(11)$ & 51 & $9(15)$ \\
$\geqslant 29$ & 36 & & & 37 & $3(5)$ \\
\hline
\end{tabular}

Values in parentheses are percentages.

or less were given surfactant in the delivery unit shortly after birth, in accordance with our clinical management protocol, while others may have received surfactant in the neonatal medical unit. The mean surfactant dose given was higher in infants of a lower gestational age $(p<0.0001)$, but this was not related to the antenatal steroid treatment in the mother $(p=0.86$; table 2$)$. Similar proportions of infants in the two groups required indomethacin for closure of a PDA, had radiological evidence of necrotising enterocolitis, developed retrolental fibroplasia (requiring laser treatment), or had culture positive sepsis. The proportion of infants in group 1 who died was nearly 1.5 times greater than that in group 2, but this difference did not reach significance $(\mathrm{p}=0.17)$. In infants with cerebral white matter lesions, $11(61 \%)$ of 18 in group 1 died compared with only five $(25 \%)$ of 20 in group $2(p=0.05)$.

One of 224 infants died within 24 hours of birth, before a cranial ultrasound scan could be carried out. All the remaining 223 infants had cranial ultrasound scans (table 2). These were reported as normal, a SEH, or an IVH in $69 \%$ of infants in group 1 and in $81 \%$ of infants in group 2 . The incidence of persistent ventriculomegaly was $6 \%$ in both the groups. By contrast, a significantly smaller proportion of infants had white matter lesions in group $2(13 \%)$ than in group $1(25 \%)$ $(\mathrm{p}=0.03)$, indicating a protective effect of antenatal steroids. For seven of the 50 infants who died, the parents gave permission for a full autopsy. The cranial ultrasound reports of "normal" brain, IVH, or ventriculomegaly were confirmed at autopsy, although no brain histology was carried out. None of these seven infants had white matter lesions on cranial ultrasound scans or at autopsy.

We compared, using univariate analysis, our clinical findings in infants who had developed white matter lesions with those who did not have similar lesions on cranial ultrasound scans (table 3). We found differences in gestational age, birth weight, exposure to a complete course of antenatal steroids, PDA, survival rate, and clinical risk index in babies (CRIB) scores. ${ }^{12}$ There was a maternal history of pre-eclampsia in eight $(21 \%)$ infants with cerebral white matter lesions and $48(26 \%)$ without cerebral white matter lesions. Pre-eclampsia had no significant effect on the incidence of cerebral white matter lesions $(p=0.68)$ and no statistically significant interactions with any of the explanatory variables (table 3 ).

Stepwise logistic regression analysis was carried out on the variables that were significant at the $5 \%$ level or less in the univariate model. This showed that gestational age $(\mathrm{p}=0.0002)$ and a complete course of antenatal steroids $(p=0.02)$ had independent effects on the incidence of cerebral white matter lesions (table 4). At a lower gestational age there is higher risk of developing white matter lesions as shown on cranial ultrasound scans. A complete course of antenatal steroids protected against cerebral white matter lesions at gestational ages of 25 weeks or less, 26-28 weeks, and 29 weeks or more.

\section{DISCUSSION}

In VLBW infants, born at a gestational age of $<31$ weeks and admitted to a regional neonatal medical unit over a two and a half year period, we have shown that exposure to a complete course of antenatal steroids halves the incidence of white matter lesions diagnosed by cranial ultrasound scan. Of the 224 infants in our study, about a third of their mothers had arrived at hospital in established labour. These deliveries had taken place before steroids could be given or before a course of steroid treatment could be completed. In the remaining two thirds, there was enough time for the mothers to complete at least one course of steroid treatment before progressing to delivery. We found that the numbers of infants in each subgroup whose mothers received one or more courses of steroid treatment were too small to investigate whether the appearance of cerebral white matter lesions was influenced by fetal exposure to repetitive courses of antenatal steroids. In VLBW infants, such white matter lesions underlie the incidence of neurodevelopmental sequelae in later childhood. We have calculated the power of our study, based on this cohort, and found it to be about $60 \%$ to detect a halving of the incidence of cerebral white matter lesions after a complete course of antenatal steroids. Increasing the size of the study group would provide more data to confirm the protective effect of antenatal steroids and not alter our conclusions.

The decision to repeat the course of antenatal steroids was an obstetric intervention commonly carried out to promote lung maturation in fetuses at risk from being born preterm, rather than protecting them from cranial abnormalities. ${ }^{2}$ The course of steroid treatment was repeated when the risk of preterm delivery had persisted or recurred after the initial treatment. The rationale for repeating courses of antenatal steroids is that changes in lung function caused by betamethasone treatment appear to be transient, ${ }^{13}$ although some effects on lung structure have been reported to persist for longer periods. ${ }^{14}$ We noted that a complete course of antenatal steroids as routinely prescribed in our hospital is not entirely in keeping with the use of betamethasone in randomised trials of the efficacy of corticosteroids in preventing neonatal respiratory distress syndrome. ${ }^{15}{ }^{16}$ In the analysis of these trials, it was noted that betamethasone is commonly given at intervals of 24 hours rather than 12-24 hours as prescribed for our mothers. The regimen in use at our hospital therefore may have made it possible for more mothers to complete a course of antenatal steroids before preterm delivery. Our criteria for an incomplete course of treatment was an interval of less than 24 hours between the first dose of steroid and delivery, and this is in keeping with the above recommendations. We did not separate the "no steroid" and the "incomplete steroid" subgroups, as there were small numbers of infants in each group and further analysis to assess outcome was not possible.

This study has certain limitations with regard to the cranial ultrasound scans. Most of the infants had two scans, but a few had one only because of either early neonatal death or transfer to a hospital near the family's home before the second scan could be carried out. We may therefore have missed lesions that may have shown up in the second scan. We therefore studied the proportions of infants who had only one scan in groups 1 and 2 and found that they were similar. Moreover, the reasons for not carrying out the second scan were similar in the two groups.

One of the authors (RA) assessed the reports available for each infant that had come from the two radiologists, who 
carry out routine cranial ultrasound scans in our neonatal medical unit, and found that their observations were consistent with one another. Another author (SR), who was blinded to the infants' clinical diagnosis, reviewed the stored copies of these scans and confirmed the findings, making the cranial ultrasound findings as certain as possible.

In the seven infants who had an autopsy, the postmortem findings confirmed the presence of normal brain, IVH, and ventriculomegaly diagnosed from cranial ultrasound scans before they died. However, none of these infants had ultrasound evidence of cerebral white matter lesions, and in none was brain histology carried out at autopsy. The postmortem findings therefore did not help us to ascertain the cause of the white matter lesions shown by cranial ultrasonography and to study neuropathological differences between echodense and echolucent lesions, as the echodensities were not reported to be transient. In previous reports, the tendency to apply the neuropathological term periventricular leucomalacia to sonographically defined echodense and/or echolucent lesions has been questioned ${ }^{17}{ }^{18}$ when the underlying neuropathology has not been investigated. The neonatal prognosis of echodense or echolucent lesions found on cranial ultrasound scans may in part be related to their antecedents, implicating hypoxia-ischaemia, inflammatory, or possibly infectious factors. Hence, white matter lesions on cranial ultrasound scans may be pathologically heterogeneous. ${ }^{18} 19$

We found that a complete course of antenatal steroids reduced neonatal mortality from $28 \%$ to $20 \%$, which is clinically a large difference. We cannot say whether this difference is due to chance, some risk factor associated with preterm birth (in those that did not receive steroids or an incomplete course of steroids), or a genuine protective effect of steroids. Many of these infants died within a few days of birth, and, because more infants in group 1 with cerebral white matter lesions died than in group 2, the proportions of survivors with cerebral white matter lesions were statistically similar in the two groups (table 2). This indicates that the steroid benefit in reducing cerebral white matter lesions is largely confined to infants who died. Antenatal steroids did not complicate maternal infections associated with chorioamnionitis or PROM, or influence the mode of delivery, the condition of the infant at birth (as assessed by CRIB scores ${ }^{12}$ ), and the incidence of culture positive neonatal sepsis. Previous concerns about the use of antenatal steroids for PROM because of their potential to cause complications from infection have not been confirmed.$^{20}$ In our study, surfactant therapy was available to all the infants in groups 1 and 2 and was not influenced by antenatal steroid exposure. We did not find a steroid effect on the incidence of PDA or necrotising enterocolitis in group 2 infants. Furthermore, the steroid advantage did not extend to SEH, IVH, or ventriculomegaly, but we cannot explain these observations. However, there is a possibility of improved lung function as a result of the steroids promoting fetal lung maturation, ${ }^{21}$ but this may not have been sufficient to enable more infants to adapt to an extrauterine existence and to survive in group 2.

As steroids are anti-inflammatory agents, they may block the action of proinflammatory cytokines, which may be a cause of cerebral white matter lesions in the most vulnerable groups of newborn infants. ${ }^{22}$ Intrauterine infection, preterm birth, and cerebral white matter lesions have been associated with an increase in endogenous synthesis of cytokines. Indeed, previous studies have reported higher concentrations of certain cytokines (interleukin-6, interleukin-1 $\beta$, and tumour necrosis factor- $\alpha$ ) in amniotic fluid because of infection. ${ }^{23}$ These cytokines are critical mediators of inflammation in the maternal uterine environment, and they provide evidence to support a causal relation between intrauterine infection and preterm delivery. ${ }^{24}$ However, when produced in excess, tumour necrosis factor- $\alpha$ is a likely cytokine for the link between intrauterine infection and cerebral white matter lesions observed in preterm infants who later develop cerebral palsy. ${ }^{25}$ Furthermore, these studies ${ }^{22-25}$ lend support to the possibility that genetic factors involved in tumour necrosis factor- $\alpha$ regulation may modify the risk of preterm labour associated with infection and the infant's risk of cerebral white matter damage. ${ }^{26}$ In our study, PROM was the only indicator of a possible intrauterine infection. PROM for a duration $>24$ hours has been associated with a greater risk of intrauterine infection ${ }^{27}$ and chorioamnionitis, with a fourfold increase in preterm birth associated with $\mathrm{PROM}^{28}$ We noted that there are more infants in group 2 than in group 1 with a maternal history of PROM for $>24$ hours. This may have occurred because the criteria for selection to group 2 included a 24 hour interval between the mother receiving the first dose of steroid and delivery. More definitive evidence linking intrauterine infection with preterm labour and cerebral white matter lesions is missing in our study.

Antenatal steroids afford considerable benefit to the preterm infant, but some caution should be exercised in prescribing repetitive courses of steroids to improve fetal outcome. In many species studied, access of maternal endogenous glucocorticoids to the fetus is low. ${ }^{29}$ Expression of $11 \beta$ hydroxysteroid dehydrogenase, in particular the type 2 isoform, in the placenta is of primary importance in excluding maternal glucocorticoids from the fetus. ${ }^{30-32}$ However, the type 2 isoform has a low affinity for exogenous glucocorticoids, ${ }^{32}$ allowing dexamethasone and betamethasone to pass rapidly from mother to fetus. A reduction in the incidence of cerebral white lesions in our study and previous reports of a reduction in other cranial ultrasound abnormalities including IVH and ventriculomegaly $^{517}$ resulting from antenatal steroids are important benefits. These cranial ultrasound abnormalities have been associated with developmental disorders in VLBW infants, including cerebral palsy, but follow up studies do not show clear evidence of cognitive deficits due to antenatal steroids in children without major disabilities. ${ }^{33}{ }^{34}$ Furthermore, a randomised study of the long term effects of antenatal dexamethasone has suggested a reduction in the incidence of cerebral palsy. ${ }^{35}$ There are, nevertheless, concerns about synthetic glucocorticoids being used extensively in fetuses at risk from being delivered preterm. The potential long term consequences of antenatal steroid exposure on brain development and the underlying mechanisms involved have been extensively reviewed. ${ }^{36}$ It is important that we should carefully consider these reports of antenatal steroid exposure in animal studies now performed in many species that have shown morphological changes in the hippocampus, permanent programming of hypothalamic-pituitary-adrenal function, and alterations in neurotransmitters and their receptors with functional consequences. ${ }^{36}$

\section{ACKNOWLEDGEMENTS}

We thank the consultant paediatricians who looked after these infants in the neonatal medical unit and the consultant radiologists who carried out the cranial ultrasound scans for allowing us to study the case notes and the cranial ultrasound reports.

\section{Authors' affiliations}

R Agarwal, M L Chiswick, Neonatal Medical Unit, St Mary's Hospital, Hathersage Road, Manchester M13 OJH, UK

S Rimmer, Department of Radiology, St Mary's Hospital

G M Taylor, Immunogenetics Laboratory, St Mary's Hospital

R J Q McNally, R D Alston, CRC Paediatric and Familial Cancer

Research Group, Royal Manchester Children's Hospital, Manchester M27 4HA, UK

S W D'Souza, Academic Unit of Child Health, University of Manchester, St Mary's Hospital

\section{REFERENCES}

1 Liggins GC, Howie RN. A controlled trial of antepartum glucocorticoid treatment for the prevention of RDS in preterm infants. Pediatrics 1972;50:515-25. 
2 Crowley P, Chalmers I, Kierse MNN. The effect of corticosteroid administration before preterm delivery: an overview of the evidence from controlled trials. Br J Obstet Gynaecol 1990;97:1 1-25.

3 Baver CR, Morrison JC, Poole WK, et al. A decreased incidence of NEC after prenatal glucocorticoid therapy. Pediatrics 1984:73:682-8.

4 Clyman RI, Ballard PL, Sniderman S, et al. Prenatal administration of betamethasone for prevention of PDA. J Pediatri 1981;98:123-6.

5 Leviton A, Dammann O, Allred EN, et al. Antenatal corticosteroids and cranial ultrasound abnormalities. Am J Obstet Gynecol

1999:181:1007-17.

6 Lorenz JM, Wooliever DE, Jetton JR, et al. A quantitative review of mortality and developmental disability in extremely preterm newborns. Arch Pediatr Adolesc Med 1998;152:425-35.

7 Vohr BR, Wright LL, Dusick AM, et al. Neurodevelopmental and functional outcomes of ELBW infants in the National Institute of Child Health and Human Development neonatal research network, 1993-1994. Pediatrics 2000;105: 1216-26.

8 Whitaker AH, Feldman JF, Van Rossem R, et al. Neonatal cranial ultrasound abnormalities in low birth weight infants: relation to cognitive outcome at six years of age. Pediatrics 1996:98:719-29.

9 Pinto-Martin JA, Riolo S, Cnaan A, et al. Cranial ultrasound prediction of disabling and non-disabling cerebral palsy at age two in a low birth weight population. Pediatrics 1995;95:249-54.

10 Dardzinski BJ, Smith SL, Towfighi J, et al. Increased plasma beta-hydroxybutyrate, preserved cerebral energy metabolism and amelioration of brain damage during neonatal hypoxia ischemia with dexamethasone pretreatment. Pediatr Res 2000;48:248-55.

11 Levene MI. Measurement of the growth of the lateral ventricles in preterm infants with real-time ultrasound. Arch Dis Child $1981 ; 56: 900-4$

12 The International Neonatal Network. The CRIB (clinical risk index for babies) scores: a trial for assessing initial neonatal risk and comparing performance of neonatal intensive care units. Lancet 1993;342:193-8.

13 Ikegami $\mathbf{M}$, Polk DH, Jobe AH, et al. Effect of interval from corticosteroid treatment to delivery on postnatal lung function in preterm lambs. J Appl Physiol 1996;80:591-7.

14 Liley H, White RT, Warr RG, et al. Regulation of messenger RNAs for the hydrophobic surfactant proteins in human lung. J Clin Invest 1989:83:1191-7.

15 Crowley P. Prophylactic corticosteroids for preterm delivery (Cochrane review). In: The Cochrane Library Issue 3. Oxford: Update Software 1999

16 National Institutes of Health Consensus Development Conference Statement. Effect of corticosteroids for fetal maturation on perinatal outcomes, February 28-March 2, 1994. Am J Obstet Gynecol $1995 ; 173: 246-52$

17 Hope PI, Gould SJ, Howard S, et al. Precision of ultrasound diagnosis of pathologically verified lesions in the brains of very preterm infants. Dev Med Child Neurol 1988;30:457-71.

18 Kuban KCK. White-matter disease of prematurity, periventricular leukomalacia, and ischemic lesions. Dev Med Child Neurol 1998;40:571-3.
19 Paneth N. Clarifying brain damage in preterm infants. J Pediatr 1999; 134:527-9.

20 Vermillion ST, Soper DE, Bland ML, et al. Effectiveness of antenata corticosteroid administration after preterm premature rupture of the membranes. Am J Obstet Gynaecol 2000;183:925-9.

21 Grover, TR, Ackerman KG, Le Cras TD, et al. Repetitive prenatal glucocorticoids increase lung endothelial nitric oxide synthase expression in ovine fetuses delivered at term. Pediatr Res 2000;48:75-83.

22 Dammann O, Leviton A. Maternal intrauterine infection, cytokines and brain damage in the preterm newborn. Pediatr Res 1997:42:1-8.

23 Yoon BY, Jun JK, Romero R, et al. Amniotic fluid inflammatory cytokines (interleukin-6, interleukin-1 $\beta$ and tumor necrosis factor- $\alpha$ ), neonatal white matter lesions and cerebral palsy. Am J Obstet Gynecol 1997; 177: 19-26.

24 Romero R, Gomez R, Ghezzi F, et al. The onset of spontaneous preterm parturition is preceded by an intense pro-inflammatory cytokine response in the human fetus. Am J Obstet Gynecol 1997;176:S3.

25 Leviton A. Preterm birth and cerebral palsy: is tumor necrosis factor the missing link? Dev Med Child Neurol 1993:35:553-8.

26 Dammann O, Durum SK, Leviton A. Modification of the infection-associated risks of preterm birth and white matter damage in the preterm newborn by polymorphisms in the tumor necrosis factor-locus? Pathogenesis 1999;1:171-7

27 Gomez R, Ghezzi F, Romero R, et al Premature labor and intraamniotic infection. Clin Perinatol 1995:22:281-342.

28 Guinn DA, Goldenburg RL, Hauth JC, et al. Risk factors for the development of preterm premature rupture of the membranes after arrest of preterm labor. Am J Obstet Gynecol 1995;173:1310-15.

29 Dalle M, Delost P. Feto-maternal production and transfer of cortisol during the last days of gestation in the guinea-pig. J Endocrinol 1979:82:43-51.

30 Edwards CRW, Benediktsson R, Lindsay RS, et al. $11 \beta$-Hydroxysteroid dehydrogenases: key enzymes in determining tissue-specific glucocorticoid effects. Steroids 1996;61:263-9.

31 Seckl JR. Glucocorticoids, feto-placental $11 \beta$-hydroxydehydrogenase type 2, and the early life origins of adult disease. Steroids 1997;62:89-94.

32 Benediktsson R, Calder AA, Edwards CRW, et al. Placental $11 \beta$-hydroxysteroid dehydrogenase: a key regulator of fetal glucocorticoid exposure. Clin Endocrinol (Oxf) 1997;46:161-6.

33 MacArthur BA, Howie RN, Dezoete JA, et al. School progress and cognitive development of 6-year old children whose mothers were treated antenatally with betamethasone. Pediatrics 1982;70:99-105.

34 Collaborative Group on Antenatal Steroid Therapy. Effect of antenatal steroid administration on the infant: long term follow-up. J Pediatr 1984:104:259-67.

35 Salokorpi T, Sajaniemi N, Hallback H, et al. Randomized study of the effect of antenatal dexamethasone on growth and development of premature children at a corrected age of two years. Acta Paediatr Scand 1997;86:294-8.

36 Mathews SG. Antenatal glucocorticoids and programming of the developing CNS. Pediatr Res 2000;47:291-300. 